\title{
Highly efficient top-down processed blue InGaN nanoscale light-emitting diodes
}

Changhee Lee ( $\nabla$ chlee7@samsung.com )

Samsung Display Co https://orcid.org/0000-0003-2800-8250

Mihyang Sheen

Yunhyuk Ko

Samsung display

Dong-uk Kim

Jongil Kim

Sungkyunkwan University

Jinho Byun

Pusan National University

Ki Young Yeon

Samsung display

Do hyung Kim

Samsung display

Jungwoon Jung

Samsung display

Jinyoung Choi

Samsung display

Ran Kim

Samsung display

Jewon Yoo

Samsung display

Inpyo Kim

Samsung display

Chanwoo Joo

Samsung display

Nami Hong

Samsung display

Joohee Lee

Samsung display

Sang Ho Jeon

Samsung display

Sang Ho Oh 
Sungkyunkwan University https://orcid.org/0000-0001-5808-7821

\section{Jaekwang Lee}

Pusan National University

\section{Nari Ahn}

Samsung display

\section{Physical Sciences - Article}

Keywords: indium gallium nitride, nanorod-LED, external quantum efficiency

Posted Date: October 28th, 2021

DOl: https://doi.org/10.21203/rs.3.rs-942933/v1

License: (9) This work is licensed under a Creative Commons Attribution 4.0 International License. Read Full License

Version of Record: A version of this preprint was published at Nature on August 3rd, 2022. See the published version at https://doi.org/10.1038/s41586-022-04933-5. 


\section{Abstract}

Demands for high-performance displays with high pixel density and picture quality are ever increasing. Indium gallium nitride (InGaN)-based micro-LEDs ( $\mu$ LEDs) are suitable for meeting such demands owing to their high efficiency, brightness, and stability. However, the poor yield of the pick-and-place technique, defect repair, and visibility of edge lines between modules limit the applications of $\mu L E D s$. Furthermore, the external quantum efficiency (EQE) decreases $(<10 \%)$ when $\mu$ LED size is reduced to less than $10 \mu \mathrm{m}$ for high pixel densities, thereby limiting the luminance. Here, we demonstrate a top-down-processed blue InGaN/GaN multiple-quantum well (MQW) nanorod-LED (nLED) can be made highly efficient as well as become an enabling technology for reducing manufacturing cost of large-screen displays. A pixel array comprising of horizontally-aligned nLEDs between pixel electrodes can be cost-effectively fabricated by applying the dielectrophoretic force to the inkjet-printed nLEDs dispersed in ink solution. To overcome size-dependent EQE reduction problem, we studied the interaction between the $\mathrm{GaN}$ surface and the surface passivation layer via various analyses and found that minimizing the point defects created during the passivation process is crucial to manufacturing high-performance nanoscale LEDs. Notably, the sol-gel method is advantageous for the passivation because $\mathrm{SiO} 2$ nanoparticles are adsorbed on the $\mathrm{GaN}$ surface, thereby minimizing its atomic interactions. The fabricated nLEDs exhibited an EQE of $20.2 \pm 0.6 \%$, the highest EQE value ever reported for the LED in the nanoscale. This work opens the way for manufacturing self-emissive nLED displays that can fully meet the industry requirements of high efficiency and brightness and low-power consumption, contributing to energy saving, carbon neutrality and mitigating climate crisis.

\section{Introduction}

Light-emitting diodes (LEDs) have been continuously making technological advances and are being used in various applications such as solid-state lightings, displays, optical data communications and photonics. ${ }^{1-3}$ In particular, the huge opportunity in the display applications such as large-screen TVs and public display products over 100 inches for high brightness and contrast are leading the recent development of micro-LEDs ( $\mu$ LEDs). ${ }^{4-6}$ Moreover, the rapid growth of the market for augmented reality (AR) and extended reality (XR) demanding high pixel density per inch (PPI) (>5000 PPI) and maintaining high luminance $\left(>10,000 \mathrm{~cd} / \mathrm{m}^{2}\right)^{7-10}$ is also accelerating a paradigm shift from a conventional liquid crystal display (LCD) to the self-emissive $\mu \mathrm{LED}$ displays.

InGaN/GaN multiple-quantum well (MQW) mLEDs are considered as a most viable technology for display applications requiring high PPI and brightness because they have the advantages of high efficiency, brightness and excellent stability. ${ }^{11,12}$ For most display applications, especially high-resolution AR displays, the LED chip size on the order of $\mu \mathrm{m}$ or sub- $\mu \mathrm{m}$ is required. ${ }^{9}$ However, the small-size $\mu$ LEDs have a major problem that the luminous efficiency decreases as the size decreases. ${ }^{13-16}$ It is mainly attributed to increased non-radiative recombination loss at the surface as the surface-to-volume ratio increases for smaller chip size. ${ }^{9,15,16}$ Although the surface recombination velocity, a key parameter for surface 
recombination, of InGaN-based materials (about $3 \times 10^{2} \sim 10^{4} \mathrm{~cm} / \mathrm{s}$ ) is orders of magnitude lower than other III-V semiconductors such as GaAs and InP, ${ }^{17}$ the Shockley-Read-Hall (SRH) recombination at surface defects becomes severe in the $\mu \mathrm{m}$ scale InGaN-LEDs. ${ }^{13-16}$ In the manufacturing processes of $\mu L E D s$, defects, impurities, and dangling bonds which act as nonradiative recombination centers are generated on the etched surface. ${ }^{9,14,16}$ It was reported that the external quantum efficiency (EQE) of blue InGaN LEDs decreases from $\sim 10 \%$ at the lateral dimension of $10 \mathrm{~mm}$ down to about $2 \sim 3 \%$ at the $1 \mathrm{~mm}$ size. ${ }^{16}$ Therefore, development of $\mu L E D$ displays with high efficiency and brightness requires for developing a technology that minimizes surface defects that occurs when manufacturing $\mu \mathrm{m}$ or sub-mmsized (nanoscale) LEDs and protects the surface with a robust passivation layer.

Development of highly efficient nanoscale LEDs can also make a breakthrough in reducing manufacturing cost of large-size display products. Current manufacturing process of $\mu \mathrm{LED}$ displays based on the 'pick-and-place' method has a high manufacturing cost because of slow tact time and low production yield. ${ }^{6}$ To overcome this technical challenge, we are developing a new cost-effective method for fabricating self-emitting nanorod LED (nLED) display ${ }^{18}$, wherein each pixel comprises of about 10 or less $\mathrm{nLEDs}$ connected in parallel. The InGaN/GaN MQW nanorods were dispersed on the substrate via inkjet printing and were horizontally-aligned on pre-patterned electrodes using dielectrophoretic force. ${ }^{18-20}$ This configuration not only increases the extraction efficiency but also provides control over the luminance by adjusting the number of nLEDs in the pixel. Moreover, the nLED display can overcome the inherent limitations of current manufacturing process of $\mu \mathrm{LED}$ displays. Compared with the timeconsuming pick-and-place method, the utilization of electric-field-assisted alignment of inkjet-printed nLEDs shortens the process time and lowers the unit $\operatorname{cost}^{18}$. Furthermore, the nLED display is less sensitive to luminance deviation due to epitaxial defects. Because many nLEDs are connected in parallel in each pixel, the differences in brightness between nLEDs are averaged across the pixel. Although the nLED display has a great potential of realizing high-performance next-generation display, the development of highly efficient nLEDs by overcoming size-dependent EQE reduction problem is a great challenge.

The sidewalls of the InGaN/GaN MQW nanorods contain various structural defects, such as surface dangling bonds, strain-relaxation-induced defects, and residual damage from the dry-etch process. ${ }^{13,16,21,22}$ It was reported that surface passivation is an effective way to reduce defect states and thereby increase the EQE. ${ }^{21-24}$ However, it is inevitable that the nLED is exposed to thermal energy, plasma, and atomic reactions during the conventional passivation processes of chemical vapour deposition (CVD) or atomic layer deposition (ALD). ${ }^{21-23}$ As a result, the fragile sidewall surfaces are susceptible to structural damages, resulting in the formation of atomic defects.

In this study, we will demonstrate that a low-temperature sol-gel process can minimize the formation of defects during the surface passivation process, leading to high-performance nanoscale LED devices. Because $\mathrm{SiO}_{2}$ nanoparticles formed after the sol-gel reaction ${ }^{25-27}$ are adsorbed to the surface, the physicochemical reactions with InGaN/GaN nanorods can be minimized. By comparing two In GaN/GaN 
nLEDs with the $\mathrm{SiO}_{2}$ passvation layer deposited by either plasma ALD or sol-gel reaction process after the top-down etching process, we found that the EQE is very sensitive and varies significantly depending on the surface passivation processs. A peak EQE of $20.2 \pm 0.6 \%$ is attained for the nLEDs with the sol-gelprocessed $\mathrm{SiO}_{2}$ passivation layer. Considering the trend of the decreasing EQE of LEDs upon size reduction (Extended Data Fig. 1), this is a remarkable achievement and offers the possibility of fabricating highly efficient nLED displays. Using extensive analysis methods auch as photoluminescence $(P L)$, cathodoluminescence $(C L)$, and electron energy-loss spectroscopy (EELS) we identified the defects that cause the performance deterioration in the top-down-processed nLEDs. When the $\mathrm{SiO}_{2}$ passivation layer is formed with an ALD process, gallium vacancy $\left(\mathrm{V}_{\mathrm{Ga}}\right)$ complex defects are created on the sidewalls of InGaN/GaN nanorods, resulting in an increase in the Shockley-Read-Hall (SRH) recombination and a decrease in the EQE. This result was cross-validated via X-ray photoelectron spectroscopy (XPS) and deep-level transient spectroscopy (DLTS).

\section{Results And Discussion}

The fabrication process and surface treatment of the nLEDs consisting of ITO/p-GaN/MQW/n-GaN are shown in Fig. $1 \mathrm{a}-\mathrm{c}$. We used commercially grown 4" epitaxial wafers, where 8 pairs of InGaN/GaN MQW LED structures were grown on a c-plane sapphire substrate. A nanorod pattern with the diameter of about $600 \mathrm{~nm}$ was constructed via nano-imprint lithography, and then it was dry etched by inductively coupled plasma reactive ion etching (ICP-RIE). Subsequently, potassium hydroxide $(\mathrm{KOH})$ wet etching was conducted to remove the sidewall damages from dry etching process. ${ }^{23,28}$ The profile of the nanorod is transformed from trapezoidal to vertical cylinder because of the high etching-barrier index of the $m$-plane surface ${ }^{29}$, and the surfaces of the nanorods become smooth (Fig. 1b). The average diameter and length of the $\mathrm{InGaN} / \mathrm{GaN}$ nanorods is $580 \mathrm{~nm}$ and $4 \mu \mathrm{m}$, respectively.

Because our pixel comprised of laterally self-aligned nLEDs, the $p$-GaN and $n-G a N$ should be isolated by an insulator. In addition, a single insulating layer is insufficient to simultaneously achieve the high efficiency, good reliability, and processability required for display products. Therefore, we applied two insulating layers on the nanorods. The inner and outer layers were for sidewall passivation and etch stopping during pixel processing, respectively. We used $\mathrm{SiO}_{2}\left(60 \mathrm{~nm}\right.$ thickness) and $\mathrm{Al}_{2} \mathrm{O}_{3}(20 \mathrm{~nm}$ thickness) as the inner and outer layers, respectively, because of their high transparency, low leakage current, and high bandgap energy ${ }^{30,31}$. Optimization of the inner insulating layer, which is directly deposited on the GaN surface, is of utmost importance for achieving highly efficient nLEDs. Even when the same insulating material is used, the LED efficiency strongly depends on the process conditions of the ALD such as the substrate temperature, process time, and use of plasma. This dependence directly corresponds to the creation of $\mathrm{V}_{\mathrm{Ga}}$ complex defects on the $\mathrm{GaN}$ surface during deposition, as will be demonstrated below.

To minimize the surface damage during the passivation process, we developed a wet-chemical process for synthesizing the $\mathrm{SiO}_{2}$ passivation layer via a sol-gel method ${ }^{25-27}$. Figure $1 \mathrm{c}$ and the inset image show 
the deposition of $\mathrm{SiO}_{2}$ and a simplified schematic of the sol-gel reaction, respectively. Performing the sol-gel process at room temperature could minimize the atomic reaction between the GaN surface and the $\mathrm{SiO}_{2}$ layer and enhance the optical properties of the nanorods by passivating the dangling bonds.

The TEM image in the inset of Fig. 1c shows a well-defined and uniform $\mathrm{SiO}_{2}$ layer on the nanorods from the top to bottom. The thickness of the layer increased linearly with an increase in the reaction time until 60 min and saturated at $23 \mathrm{~nm}$ owing to the limited quantity of sol particles (Extended Data Fig. 2). In addition, upon doubling the thickness of $\mathrm{SiO}_{2}$ layer up to $46 \mathrm{~nm}$ by repeating the sol-gel reaction, the PL intensity increased because the structural stress and surface defects of the $\mathrm{SiO}_{2}$ layer decreased with an increase in the thickness ${ }^{32}$. To evaluate the passivation uniformity, we recorded the PL spectra from three different positions on the 4" wafer (Extended Data Fig. 2). We found that the variation in the PL intensity with respect to the position is minor.

The PL images and the fluorescence excitation-emission spectra (Fig. 1d) and the panchromatic $(\lambda=300$ - $700 \mathrm{~nm}$ ) CL images (Fig. 1e) are compared for the nanorods passivated by the different methods; conventional plasma enhanced $\mathrm{ALD}$-deposited $\mathrm{SiO}_{2}$ (left) and sol-gel-deposited $\mathrm{SiO}_{2}$ (right). When the sol-gel process is applied for the $\mathrm{SiO}_{2}$ passivation, the enhancement of the blue emission over yellow emission is clearly observed in Fig. 1d. The intensity of blue emission of the sol-gel $\mathrm{SiO}_{2}$-passivated nanorods is approximately 13 times higher than that of $A L D \mathrm{SiO}_{2}$-passivated nanorods (Fig. 1f). In addition, the panchromatic $C L$ images of the individual nanorod LEDs in Fig. 1e clearly visualizes that the existence of the non-radiative recombination region depending on the passivation method. ${ }^{33}$ The outer rim corresponds to the yellow emission from defects including $\mathrm{V}_{\mathrm{Ga}}$-related defects ${ }^{34,35}$, and the inner circle corresponds to the blue emission of the MQW regions. In the case of $\mathrm{ALD} \mathrm{SiO}_{2}$-passivated nanorods (Fig. 1e. left), the outside of the MQWs is dark owing to the non-radiative recombination centre (NRC). Additionally, the nanorods exhibit significant differences in brightness because of the different positions of the NRC. However, for the sol-gel $\mathrm{SiO}_{2}$-passivated nanorods (Fig. 1e. right), the overall brightness is uniform and the above-mentioned abnormal light emission is absent. Furthermore, the reduced carrier lifetime of the sol-gel $\mathrm{SiO}_{2}$-passivated nanorods verifies that the surface defects of $\mathrm{GaN}$ are reduced than that of the ALD-deposited $\mathrm{SiO}_{2}$ (Fig. 1g).

Figure 2a shows the EL and PL composite images of a single nLED within a pixel according to the passivation method used. The pixel is composed of the nanorods connected in parallel with transparent conductive metals ${ }^{18}$. Because the EL of the laterally aligned $\mathrm{nLED}$ is emitted along the radial and longitudinal directions owing to waveguide reflection, a bright blue and a relatively dark emission are observed at the top and bottom of the nanorods, respectively. According to the light extraction calculation for our pixel design, the total light extraction efficiency (LEE) is approximately $25 \%$, and it comprises $71 \%$ radial emission and $17 \% \mathrm{p}$-side and $10 \% \mathrm{n}$-side waveguide emission. The EL intensity profiles obtained from a region horizontally across the top of the nLEDs confirm that the EL intensity of the sol-gel $\mathrm{SiO}_{2}-$ passivated nanorod is higher than that of the ALD $\mathrm{SiO}_{2}$-coated nanorod (Fig. 2b). 
Figure 2c shows the EQE curves for the nLEDs for each surface passivation type. Each curve is obtained from a 60-pixel array, and each pixel comprises an average of 6 and 9 nLEDs for ALD $\mathrm{SiO}_{2}$ - and sol-gel $\mathrm{SiO}_{2}$ passivation, respectively. The difference in the number of the $\mathrm{nLEDs}$ per pixel originates from the self-aligned process dispersion. The average values of the peak EQE of the sol-gel- and $\mathrm{ALD} \mathrm{SiO}_{2}-$ deposited nLEDs are 20.2\% (standard deviation = 0.6\%) and 8.9\% (standard deviation $=0.1 \%$ ), respectively. Furthermore, the IQE, which was obtained by dividing the EQE by the calculated LEE, was $81 \%$ and $36 \%$ for the sol-gel $\mathrm{SiO}_{2}$ - and $\mathrm{ALD} \mathrm{SiO}_{2}$-deposited nLEDs, respectively. The high EQE of the solgel $\mathrm{SiO}_{2}$-deposited $\mathrm{nLED}$ is remarkable because it is higher than the best EQE value of the $\mu$ LED structure even at a larger diameter ${ }^{16}$. The primary reason for such a high EQE is the decrease in the GaN surface damage in the sol-gel $\mathrm{SiO}_{2}$-deposited $\mathrm{nLED}$, as confirmed by the longer carrier lifetime in PL and decreased NRC region in CL in Fig. 1. Furthermore, this is observed in the $\mathrm{J}-\mathrm{V}$ electrical characteristics of the LEDs.

The sol-gel $\mathrm{SiO}_{2}$-deposited nLEDs exhibit a lower leakage current than that of the $\mathrm{ALD} \mathrm{SiO}_{2}-$ deposited nLEDs at below-threshold voltages (Fig. 2d, e) owing to the parallel resistance component attributed to sidewall damage. This is consistent with the decrease in the ideality factor of sol-gel $\mathrm{SiO}_{2}-$ deposited nanorods compared with that of $\mathrm{ALD} \mathrm{SiO}_{2}$-deposited nanorods (Fig. 2f). Generally, the ideality factor of the LED structure changes significantly depending on its epi structure ${ }^{36}$; however, we have proven that the ideality factor can also be changed significantly by the passivation method. The decrease in the ideality factor of sol-gel $\mathrm{SiO}_{2}$-deposited nanorods indicates a decrease in the effective $\mathrm{SRH}$ recombination in the $A B C$ model ${ }^{14,37}$, thereby increasing the radiative recombination and internal quantum efficiency to over $80 \%$.

The significant change in the electrical and optical properties depending on the method of deposition of the side-wall insulating layer is closely related to the defects generated during the passivation process. These defects could prevent the passivation of the GaN surface dangling bonds, thereby degrading the device performance. Therefore, understanding the phenomenon occurring at the interface and controlling it are the key to manufacturing nLEDs with outstanding performance.

Figure 3 shows the analyses results of the interface between the sidewall of the nanorods and the insulator. First, we traced the evolution of the morphology and atomic structure of the sidewall in the MQW region after each fabrication step via high-angle annular dark-field scanning transmission electron microscopy (HAADF-STEM) imaging (Fig. 3a). After the dry etching of the epi layer, the nanorod developed a trapezoidal shape owing to the impact-energy difference on the surface. The white arrows indicate the amorphous region at the sidewall, which formed owing to successive ion bombardments. However, after $\mathrm{KOH}$ wet etching, the nanorod developed an inverted trapezoidal shape because the etch rate of $p$-GaN is slower than that of $n-\mathrm{GaN}^{38}$. Additionally, the semi-polar plane and $m$-plane appear alternately, resulting in a staircase-like sidewall morphology of the InGaN QWs. The white arrow indicates the native oxide layer on the nanorod surface. When the $\mathrm{SiO}_{2}$ layer is deposited via plasma enhanced ALD, the plasma creates point defects, such as nitrogen vacancies $\left(V_{N}\right)$, nitrogen interstitials $\left(N_{i}\right)$, nitrogen 
split interstitials $(\mathrm{N}-\mathrm{N})_{\mathrm{N}}, \mathrm{V}_{\mathrm{Ga}}$, and $\mathrm{V}_{\mathrm{Ga}}$ complexes ${ }^{39,40}$, resulting in the amorphization of the surface of the nanorods. This amorphous region is observed on the entire surface of the GaN nanorod; however, it is predominantly observed on the semi-polar facet of the InGaN QWs (yellow arrows), probably owing to the high density of dangling bonds. With an increase of the thickness of the $\mathrm{SiO}_{2}$ layer from $2 \mathrm{~nm}$ to $60 \mathrm{~nm}$ (Extended Data Fig. 3e), the penetration depth of the plasma-induced amorphization increases to $4 \mathrm{~nm}$ at the InGaN QWs. However, when sol-gel $\mathrm{SiO}_{2}$ is deposited on the nanorods, there is no amorphous region formed at the interface in addition to the deposited $\mathrm{SiO}_{2}$.

Figure $3 \mathrm{~b}, \mathrm{c}$ and Extended Data Fig. $4 \mathrm{~b}-\mathrm{g}$ show the XPS results. The core level spectra of $\mathrm{Ga} 3 \mathrm{~d}$ could be deconvoluted to the $\mathrm{Ga}-\mathrm{N}, \mathrm{Ga}-\mathrm{O}$, and $\mathrm{Ga}-\mathrm{Ga}$ bonds. The $\mathrm{Ga} 3 \mathrm{~d}$ state ratios (Fig. $3 \mathrm{~b}$ ) clearly indicate that the $\mathrm{Ga}-\mathrm{O}$ bonds were the highest in the ALD $\mathrm{SiO}_{2}$-coated nanorods and the lowest in the sol-gel $\mathrm{SiO}_{2}-$ deposited nanorods. Owing to the plasma-induced $\mathrm{GaN}$ defects, such as $\mathrm{V}_{\mathrm{N}}$ and $\mathrm{O}_{\mathrm{N}}$, the $\mathrm{Ga}-\mathrm{O}$ bonds increased, whereas the $\mathrm{Ga}-\mathrm{N}$ bonds decreased. Furthermore, comparing the $\mathrm{Si} 2 \mathrm{p}$ core level spectrum of sol-gel $\mathrm{SiO}_{2}$ and ALD $\mathrm{SiO}_{2}$ (Extended Data Fig. 4e), the latter exhibits oxygen deficiency because of oxygen atoms contributing to the $\mathrm{Ga}-\mathrm{O}$ bond. Moreover, we obtained electron spin resonance (ESR) spectra to analyse the concentration of the $(\mathrm{N}-\mathrm{N})_{\mathrm{N}}$ defects (Fig. 3d, e), which are known as ambipolar defects, i.e., with a deep acceptor in $n$-GaN and deep donor in $p$-GaN ${ }^{41}$. The $(\mathrm{N}-\mathrm{N})_{\mathrm{N}}{ }^{0}$ defects decreased after the wet etching of the rods and increased after $\mathrm{ALD} \mathrm{SiO}_{2}$ deposition. Because the defect concentrations are calculated by dividing the spins by the total weight of the nanorods and considering that most of the defects are concentrated at the nanorod surface, the actual differences in the defect concentrations of the nanorods after each fabrication step will be greater. Additionally, the $(\mathrm{N}-\mathrm{N})_{\mathrm{N}}{ }^{0}$ defects in the sol-gel $\mathrm{SiO}_{2}$-coated rods were fewer than those in the wet-etched rod, demonstrating the passivation of surface dangling bonds without generating excess defects.

We also carried out DLTS analysis on a bulk LED chip and ALD $\mathrm{SiO}_{2}$-coated nLED-array chip (Extended Data Fig. 5). In the bulk LED chip, the main defects are electron traps owing to $V_{N}$ and $N_{i}$ with activation energies of 0.56 and $0.67 \mathrm{eV}$, respectively ${ }^{42}$. In the nLED-array chip, although the electron trap concentration is similar to that of the bulk LED chip, five types of hole traps $(0.14,0.39,0.51,0.56$, and $0.93 \mathrm{eV}$ ) are observed, and the defect concentration is greater than $10^{15} \mathrm{~cm}^{-3}$ for the deepest defect level $\left(\mathrm{E}_{\mathrm{v}}+0.93 \mathrm{eV}\right.$ ). $\mathrm{V}_{\mathrm{Ga}}$-related complexes (complexes of $\mathrm{V}_{\mathrm{Ga}}$ and oxygen that replace nitrogen sites, i.e. $\mathrm{V}_{\mathrm{Ga}}{ }^{-}$ $\mathrm{O}_{N}, \mathrm{~V}_{\mathrm{Ga}}-\mathrm{O}_{\mathrm{N}}-2 \mathrm{H}$ ) are reportedly responsible for these hole traps ${ }^{43,44}$. According to previous reports, ${ }^{38,45}$ these $\mathrm{V}_{\mathrm{Ga}}$ complexes increase the $\mathrm{SRH}$ coefficient, thereby increasing the non-radiative recombination rate and significantly decreasing the IQE of optoelectronic devices.

Figure 4 shows a detailed comparison of two representative EEL spectra obtained from the bulk and the surface region of the InGaN QWs. For the bulk InGaN, the N-K energy-loss near-edge structure (ELNES) can be decomposed into four contributions, denoted by $A, B, C$, and $D^{46}$. For the InGaN passivated by solgel $\mathrm{SiO}_{2}$, the observed spectral features of N-K ELNES are almost similar for the bulk and surface regions (Top panel in Fig 4b). Compared with those of InGaN passivated via the sol-gel method, pronounced 
changes are observed in the spectral features of the N-K ELNES (red line) of the surface region of InGaN passivated by plasma enhanced ALD: the appearance of the small peak between the second and third peak, and the chemical shift of the third peak, labelled as C, to a lower energy (red shift).

To theoretically verify the observed spectral features of the N-K ELNES, density functional theory (DFT) calculations were performed by considering the $\mathrm{V}_{\mathrm{Ga}}-\mathrm{O}_{\mathrm{N}}-2 \mathrm{H}$ complex in a 100-atom-based $3 \times 4 \times 2 \mathrm{GaN}$ supercell, which corresponds to the most energetically favourable $V_{G a}$ point defect complexed with $O$ and $\mathrm{H}^{44}$. The major changes caused by the $\mathrm{V}_{\mathrm{Ga}}-\mathrm{O}_{\mathrm{N}}-2 \mathrm{H}$ complex are that the third peak (C) at approximately $405 \mathrm{eV}$ (bottom-most panel of Fig. $4 \mathrm{~b}$ ) is shifted toward a lower energy by approximately $0.4 \mathrm{eV}$, and a small shoulder peak appears between $\mathrm{B}$ and $\mathrm{C}$ at approximately $403 \mathrm{eV}$, which is consistent with the experimental measurements. The red shift and the formation of the shoulder peak suggest that the $\mathrm{V}_{\mathrm{Ga}}{ }^{-}$ $\mathrm{O}_{\mathrm{N}}-2 \mathrm{H}$ complex locally breaks the wurtzite symmetry by changing the relative distance between the $\mathrm{Ga}$ and $\mathrm{N}$ atoms and then modifying the hybridization between the $\mathrm{Ga}$ and $\mathrm{N} 2 \mathrm{p}$ states, resulting in a chemical shift of the $\mathrm{N}-\mathrm{K}$ edge and the formation of a shoulder peak. Therefore, we conclude that the $\mathrm{V}_{\mathrm{Ga}}-\mathrm{O}_{\mathrm{N}}-2 \mathrm{H}$ complex is the dominant defect generated in the sidewalls of the nanorods during the plasma $\mathrm{ALD}$ of $\mathrm{SiO}_{2}$, wherein a hydrogen-containing precursor is used. According to a report ${ }^{44}$, the $\mathrm{V}_{\mathrm{Ga}}{ }^{-}$ $\mathrm{O}_{\mathrm{N}}-2 \mathrm{H}$ complex dominantly causes $\mathrm{SRH}$ recombination in the InGaN QWs system, and it significantly decreases the IQE of the nLEDs owing to the relaxation of the biaxial stress at the sidewall.

Although the sol-gel method provides superior opto-electrical properties, it has a few disadvantages such as the presence of residual reaction by-products, which have to be overcome to achieve good reliability. Therefore, suitable post-treatments need to be conducted to complete the reaction and eliminate the reaction residue. We have found that this can be achieved while maintaining the high EL by baking the sol-gel $\mathrm{SiO}_{2}$ film (Extended Data Fig. 6). With these preliminary results, research on further improvement via process optimization is currently under way.

In concluion, we developed highly efficient top-down-processed nLED pixels via sol-gel $\mathrm{SiO}_{2}$ passivation. In each pixel, the nanorods were aligned on pre-patterned electrodes using the dielectrophoretic force and connected in parallel via transparent electrodes. We achieved a peak EQE of $20.2 \%$ with a low leakage current at below-threshold voltages and an ideality factor of 1.54. Additionally, by performing various analyses on the surface and the interface between the nanorods and the insulating layer via DLTS, XPS, ESR, and STEM-EELS, we demonstrated that passivation via conventional plasma ALD induces amorphization at the InGaN QW surfaces and creates point defects on the sidewalls of GaN nanorods, including high concentrations of ambipolar $\mathrm{N}$ split interstitials and $\mathrm{V}_{\mathrm{Ga}}$-complexes, which increases the $\mathrm{SRH}$ recombination and generates an NRC region at the InGaN sidewall of the nanorods. Moreover, the sol-gel process is advantageous for insulating the $\mathrm{GaN}$ surfaces because the $\mathrm{SiO}_{2}$ nanoparticles are adsorbed on the $\mathrm{GaN}$ surface after the sol-gel reaction. Thus, the atomic interaction with the GaN surface is minimized and only dangling bonds of the surfaces are passivated, resulting in a low leakage current, decrease in the NRC regions, and high EQE of over $20 \%$. We strongly believe that our findings could accelerate the implementation of nLEDs in next-generation displays. 


\section{References}

1. Schubert, E. F. Light-Emitting Diodes (Cambridge University Press, Cambridge, 2006).

2. Lin, J. Y. \& Jiang, H. X. Development of microLED. Appl. Phys. Lett. 116, 100502 (2020).

3. Ren, A. et al. Emerging light-emitting diodes for next-generation data communications. Nat Electron 4, 559-572 (2021).

4. Huang, Y. et al. Mini-LED, Micro-LED and OLED displays: present status and future perspectives. Light Sci. Appl. 9, 105 (2020).

5. Biwa, G. et al. Technologies for the Crystal LED display system. J. Soc. Inf. Display 29, 435-445 (2021).

6. Virey, E. H. \& Baron, N. 45-1: Status and prospects of microLED displays, SID Symp. Dig. Tech. Pap. 49, 593-596 (2018).

7. Templier, F. GaN-based emissive microdisplays: A very promising technology for compact, ultra-high brightness display systems. J. Soc. Inf. Display 24, 669-675 (2016).

8. Zhang L, et al. 31.1: Invited Paper: Monochromatic active matrix micro-LED microdisplays with $>5,000$ dpi pixel density fabricated using monolithic hybrid integration process. SID Symp. Dig. Tech. Pap. 49, 333-336 (2018).

9. Liu, Z. et al. Micro-light-emitting diodes with quantum dots in display technology. Light Sci. Appl. 9, 83 (2020).

10. Kawanishi $\mathrm{H}$, et al. High-resolution and high-brightness full-colour "Silicon Display" for augmented and mixed reality. J. Soc. Inf. Display 29, 57-67 (2021).

11. Wierer Jr., J. J. \& Tansu, N. III-Nitride Micro-LEDs for Efficient Emissive Displays, Laser Photonics Rev. 13, 1900141 (2019).

12. Jiang H. X. \& Lin, J. Y. Nitride micro-LEDs and beyond - a decade progress review, Opt. Express 21, A475 (2013).

13. Olivier, F. et al. Influence of size-reduction on the performances of GaN-based micro-LEDs for display application. J. Lumin. 191, 112-116 (2017).

14. Hwang, D. et al. Sustained high external quantum efficiency in ultrasmall blue III-nitride micro-LEDs, Appl. Phys. Express 10, 032101 (2017)

15. Konoplev, S. S., Bulashevich, K. A. \& Karpov, S. Y. From large-size to micro-LEDs: Scaling trends revealed by modeling, Phys. Status Solidi A 215, 1700508 (2018)

16. Smith, J. M. et al. Comparison of size-dependent characteristics of blue and green InGaN microLEDs down to $1 \mathrm{~mm}$ diameter. Appl. Phys. Lett. 116, 071102 (2020).

17. Bulashevich, K. A. \& Karpov, S. Y. Impact of surface recombination on efficiency of III-nitride lightemitting diodes. Phys. Status Solidi RRL 10, 480-484 (2016).

18. Sung, Y. G. Micro-led element for horizontally-aligned assembly, method for manufacturing same, and horizontally-aligned assembly comprising same, US20170317228A1 (2017). 
19. Park, H., Kim, B.-J. \& Kim, J. Electroluminescence from InGaN/GaN multiquantum-wells nanorods light-emitting diodes positioned by non-uniform electric fields. Opt. Express 20, 25249-25254 (2012).

20. Park, H. et al. Horizontally assembled green InGaN nanorod LEDs: scalable polarized surface emitting LEDs using electric-field assisted assembly. Sci. Rep. 6, 28312 (2016).

21. Yang, C. et al. Enhancement in Light Extraction Efficiency of GaN-Based Light-Emitting Diodes Using Double Dielectric Surface Passivation. Opt. Photonics J. 2, 185-192 (2012)

22. Wong, M. S. et al. High efficiency of III-nitride micro-light-emitting diodes by sidewall passivation using atomic layer deposition. Opt. Express 26, 21324-21331 (2018).

23. Wong, M. S. et al. Size-independent peak efficiency of III-nitride micro-light-emitting-diodes using chemical treatment and sidewall passivation. Appl. Phys. Express 12, 097004 (2019).

24. Ley, R. T. et al. Revealing the importance of light extraction efficiency in InGaN/GaN microLEDs via chemical treatment and dielectric passivation. Appl. Phys. Lett. 116, 251104 (2020).

25. Dubey, R., Rajesh, Y. \& More, M. Synthesis and characterization of $\mathrm{SiO}_{2}$ nanoparticles via sol-gel method for industrial applications. Materials Today: Proceedings 2, 3575-3579 (2015).

26. Azlina, H., Hasnidawani, J., Norita, H. \& Surip, S. Synthesis of SiO2 nanostructures using sol-gel method. Acta Phys Pol A 129, 842-844 (2016).

27. Zhang, W. et al. Preparation of $\mathrm{SiO}_{2}$ anti-reflection coatings by sol-gel method. Energy Procedia 130, $72-76$ (2017).

28. Lai, Y.-Y. et al. The study of wet etching on GaN surface by potassium hydroxide solution. Res. Chem. Intermed. 43, 3563-3572 (2017).

29. Peñalba, M., Juaristi, J., Zarate, E., Arnau, A. \& Bauer, P. Electronic stopping power of $\mathrm{Al}_{2} \mathrm{O}_{3}$ and $\mathrm{SiO}_{2}$ for H, He, and N. Phys. Rev. A 64, 012902 (2001).

30. Wilk, G. D., Wallace, R. M. \& Anthony, J. High-K gate dielectrics: Current status and materials properties considerations. J. Appl. Phys. 89, 5243-5275 (2001).

31. Jeong, B. G. et al. Colloidal spherical quantum wells with near-unity photoluminescence quantum yield and suppressed blinking. ACS Nano 10, 9297-9305 (2016).

32. Chen, W. et al. Free charges versus excitons: photoluminescence investigation of InGaN/GaN multiple quantum well nanorods and their planar counterparts. Nanoscale 10, 5358-5365 (2018).

33. Koyama, H. Cathodoluminescence study of $\mathrm{SiO}_{2}$. J. Appl. Phys. 51, 2228-2235 (1980).

34. Reshchikov, M. A. \& Morkoç, H. Luminescence properties of defects in GaN. J. Appl. Phys. 97, 061301 (2005)

35. Liu, B. et al. Origin of yellow-band emission in epitaxially grown GaN nanowire arrays. ACS Appl. Mater. Interfaces 6, 14159-14166 (2014).

36. Zhu, D. et al. The origin of the high diode-ideality factors in GalnN/GaN multiple quantum well lightemitting diodes. Appl. Phys. Lett. 94, 081113 (2009). 
37. Tian, P. et al. Size-dependent efficiency and efficiency droop of blue InGaN micro-light emitting diodes. Appl. Phys. Lett. 101, 231110 (2012).

38. Zhuang, D. \& Edgar. J. H. Wet etching of GaN, AlN, and SiC: a review. Mater. Sci. Eng. R Rep. 48, 1-46 (2005).

39. Foster, G. M., et al. Recovery from plasma etching-induced nitrogen vacancies in p-type gallium nitride using UV/O3 treatments. Appl. Phys. Lett. 117, 082103 (2020)

40. Kato, M. et al. Characterization of plasma etching damage on p-type GaN using Schottky diodes. J. Appl. Phys. 103, 093701 (2008).

41. Von Bardeleben, H. J., et al. Identification of the nitrogen split interstitial $(\mathrm{N}-\mathrm{N})_{\mathrm{N}}$ in GaN. Phys. Rev. Lett. 109, 206402 (2012).

42. Reshchikov, M. A. et al. Evaluation of the concentration of point defects in GaN. Sci. Rep. 7, 9297 (2017).

43. Wickramaratne, D. et al. Defect identification based on first-principles calculations for deep level transient spectroscopy. Appl. Phys. Lett. 113, 192106 (2018).

44. Dreyer, C. E. et al. Gallium vacancy complexes as a cause of Shockley-Read-Hall recombination in IIInitride light emitters. Appl. Phys. Lett. 108, 141101 (2016).

45. Lyons, J. L. \& Van de Walle, C. G. Computationally predicted energies and properties of defects in GaN. NPJ Comput. Mater. 3, 1-10 (2017).

46. Mizoguchi, T. et al. First-principles calculations of ELNES and XANES of selected wide-gap materials: dependence on crystal structure and orientation. Phys. Rev. B 70, 045103 (2004).

\section{Methods}

\section{Fabrication of top-down processed nLEDs}

We used commercially grown 4" epitaxial wafers, which possessed 8 pairs of InGaN/GaN multiplequantum well (MQW) LED structures on c-plane sapphire substrates. An indium-tin-oxide (ITO) layer (110 $\mathrm{nm}$ thick) was deposited on top of the $p$-type $\mathrm{GaN}$ to obtain a $p$-type transparent conducting metal. Nanoimprint lithography was performed to fabricate a rod-like pattern using a $\mathrm{SiO}_{2}$-based hard mask on the ITO layer, and this pattern was dry etched via inductively coupled plasma-reactive-ion etching (ICP-RIE). Subsequently, potassium hydroxide $(\mathrm{KOH})$ wet etching was conducted to remove the surfaces of the mesa that were damaged during the dry etching process. The average diameter and length of the fabricated $\mathrm{GaN}$ rods were $580 \mathrm{~nm}$ and $4 \mu \mathrm{m}$, respectively.

\section{Fabrication of sol-gel $\mathrm{SiO}_{2}$ layer on the nLEDs}

Tetraethyl orthosilicate (TEOS, Sigma-aldrich, 98\%) was used as a precursor for the synthesis of a $\mathrm{SiO}_{2}$ passivation layer on the nanorods surface. A schematic of the entire sol-gel process is shown in Fig. 1c. First, $210 \mathrm{ml}$ of ethanol (EtOH, Sigma-aldrich, anhydrous) and $290 \mathrm{ml}$ of DI water were mixed in a three- 
necked flask. The entire 4" wafer with nanorods was dipped in this solution. Then, $0.8 \mathrm{~g}$ of cetyltrimethylammonium bromide (CTAB, Sigma-aldrich, $\geq 98 \%$ ) was dissolved in the solution at room temperature to form micelles. After stirring with $500 \mathrm{rpm}$ the above mixture for $5 \mathrm{~min}, 2.5 \mathrm{ml}$ of an aqueous ammonia solution (Sigma-aldrich, 28 30\%) and $1.25 \mathrm{ml}$ of TEOS were sequentially added dropwise into the reactants. The resulting mixture was then continuously stirred at $25^{\circ} \mathrm{C}$ with $500 \mathrm{rpm}$ for $2 \mathrm{~h}$ and washed the wafer with DI water and EtOH.

\section{PL analysis of nLEDs in the wafer and pixel structure}

2D steady-state PL spectra were obtained using a FluoreMax-4 spectrofluorometer (Horiba) with a $150 \mathrm{~W}$ ozone-free xenon arc lamp as the excitation source. Steady-state PL/EL images and a two-photon spectrum of GaN rods were obtained using a modified confocal microscope (Leica TCS SP8). The excitation sources were an enhanced mercury lamp and a Ti:sapphire laser (Chameleon Vision) producing $140 \mathrm{fs}$ pulses at a repetition rate of $80 \mathrm{MHz}$ for the steady-state PL images and two-photon spectrum, respectively. An excitation wavelength of $810 \mathrm{~nm}$ was used for the InGaN MQWs. The lamp and laser were focused through a dry objective (NA 0.8). The PL decay traces and fluorescence lifetime images were obtained via another modified confocal microscope (Leica TCS SP8) with PicoHarp 300 (Picoquant) by using the time-correlated single-photon counting technique. They were obtained by the one-photon excitation of a $405 \mathrm{~nm}$ diode laser at a repetition rate of $625 \mathrm{kHz}$. The objective lens was the same as that used in the steady-state PL measurements. All PL and EL measurements were performed at at room temperature between $20-25^{\circ} \mathrm{C}$ in a dark room.

\section{Quantification of defect concentrations via ESR}

ESR measurements were performed using a X-band Bruker ELEXSYS E500 II spectrometer. A helium gasflow cryostat was used at an operating temperature of $6 \mathrm{~K}$. The $\mathrm{GaN}$ rod powder samples were placed in a quartz sample tube with a diameter of $4 \mathrm{~mm}$ and held in a microwave cavity. The experimental ESR settings were as follows: microwave power $-0.002 \mathrm{~mW}$; sweep width-1000 G; modulation frequency-100 $\mathrm{kHz}$; modulation amplitude-5 G; sweep time-200 s; number of points-2048; number of scans -8 . The spin density was calculated using the SpinCount \& Spinfit software.

\section{Surface state analysis via XPS}

XPS data were obtained using an ESCALAB 250Xi electron spectrometer (Thermo Fisher Scientific, East Grinstead, U.K.) with a monochromatic Al-Ka X-ray $(1486.7 \mathrm{eV})$ source and a spot size of $400 \mu \mathrm{m}$. Atomic concentrations were determined from the survey spectra $(0-1350 \mathrm{eV}, 100 \mathrm{eV}$ detector pass energy, $1 \mathrm{eV}$ step size, $50 \mathrm{~ms}$ dwell time, 2 scans). Furthermore, high-resolution spectra were acquired (20 eV detector pass energy, $0.1 \mathrm{eV}$ step size, $50 \mathrm{~ms}$ dwell time, 10 scans) and the Thermo Fisher Scientific Avantage software was employed for peak fitting; the Smart background option was used. The spectrometer was calibrated using the $\mathrm{C} 1 \mathrm{~s}$ peak as a reference point $(284.8 \mathrm{eV})$, which is in excellent agreement with the average values provided by the National Institute of Standards and Technology (NIST). 


\section{Trap-density analysis via DLTS}

All the DLTS measurements in this study were conducted by using a DLS-1100 (SEMILAB, Hungary) system in the temperature range of $80-600 \mathrm{~K}$. The chips in which approximately 10,000 nanorods were $\mathrm{p}$ $\mathrm{n}$ aligned in an area of $500 \mu \mathrm{m}^{2}$ were loaded in the DLTS chamber at a vacuum of $1 \times 10^{-2}$ torr. The measurement conditions were a temperature scan of $100 \mathrm{mK} / \mathrm{sec}$ and a pulse voltage of $2 \mathrm{~V}$ to facilitate the formation of the depletion region in the nanorod device.

\section{Measurement of electrical properties}

External quantum efficiency (EQE) was calculated from the spectra obtained for test element group (TEG) cells using a CAS-140CT Array Spectroradiometer (Instrument Systems, Konica Minolta, Japan). Furthermore, the current density-voltage $(\mathrm{J}-\mathrm{V})$ curves for single pixels in TEG cells were recorded using a Keithley 2400 source measurement unit instrument (Keithley instrument, USA).

\section{Observation of surface atomic structure via TEM}

We used an aberration-corrected STEM system (Themis Z G3, Thermo Fisher Scientific) with an accelerated electron beam of $300 \mathrm{kV}$ to observe the atomic structure of the sidewall region. The nanorods were directly dispersed on lacey carbon grids to prevent damage to $\mathrm{Ga}$ ions during FIB milling of the specimen. Furthermore, the insulating layer was intentionally deposited with a thickness of only $2 \mathrm{~nm}$ for electron transparency. To observe the interface between the nanorods and the $\mathrm{SiO}_{2}$ layer, we performed STEM-EELS (JEM-ARM200F, JEOL) on an FIB specimen with $60 \mathrm{~nm}$ thick $\mathrm{SiO}_{2}$-deposited nanorods. Further, we cleaned the specimen using an Ar-ion beam to remove the areas that sustained Ga-ion damage during FIB.

\section{Electron energy-loss spectroscopic analysis}

EELS calculations were performed based on the density functional theory implemented in VASP ${ }^{47}$. The projector augmented-wave method was used to mimic the ionic functional ${ }^{48}$, whereas the PerdewBurke-Ernzerhof approximation was employed for the electron exchange-correlation functional ${ }^{49}$. Plane waves were included up to an energy cut-off of $400 \mathrm{eV}$. We used a $9.0 \times 11 \times 11 \AA$ supercell to consider defect complexes. The Brillouin zone for the supercell calculation was sampled using a $2 \times 2 \times 2 \mathrm{k}$-point grid. lonic and electronic relaxations were performed by applying a convergence criterion of $5 \times 10^{-2} \mathrm{eV} / \AA$ per ion and $10^{-5} \mathrm{eV}$ per electronic step. The super-cell core-hole method was employed to obtain the EEL spectra of $\mathrm{GaN}^{50}$. The core electron of the oxygen atom was removed from the core and added to the valence/conduction bands, resembling the final state of the excitation process (also referred to as finalstate approximation). We used a constant Lorentzian broadening of $0.5 \mathrm{eV}$ to replicate the experimental broadening.

[47] G. Kresse and J. Furthmüller, Phys. Rev. B 54, 11169 (1996) 
[48] P. E. Blöchl, Phys. Rev. B 50, 17953 (1994)

[49] J. P. Perdew, K. Burke, and M. Ernzerhof, Phys. Rev. Lett. 77, 3865 (1996)

[50] F. Karsai, M. Humer, E. Flage-Larsen, P. Blaha, and G. Kresse, Phys. Rev. B 98, 235205 (2018)

\section{Declarations}

Acknowledgements: We thank J.K.L and S.H.O for their assistance with the electron microscopy analyses to understand the behaviour of the interface between the insulator and the $\ln \mathrm{GaN}$ nanorods and discussions.

Author contributions: D.K. and J.Y. fabricated the nLEDs and the pixels using the nanorods. Y.K., I.K., C.J., and N.H. synthesized the sol-gel $\mathrm{SiO}_{2}$ layer on the nanorods. J.K. and J.B. analysed and interpreted the STEM-EELS data. K.Y., D.K., J.J., J.C. and R.K. carried out the PL, DLTS, ESR, and XPS analyses. J.L. and S.J. calculated the defect levels of GaN. S.H.O. and J.L. contributed to the interpretation of the experimental results and calculated data. N.A. and C.L. supervised the research and coordinated the work. M.S. contributed to the experiments and analyses, and M.S. and C.L. wrote the manuscript with input from all other authors.

Competing interests: The authors declare no competing interests.

Additional information: Correspondence and requests for materials should be addressed to M.H.S (mihyang.sheen@samsung.com).

Reprints and permissions information is available at www.nature.com/reprints

Data availability: All data generated or analysed during this study are included in the paper and its Supplementary Information files.

\section{Figures}




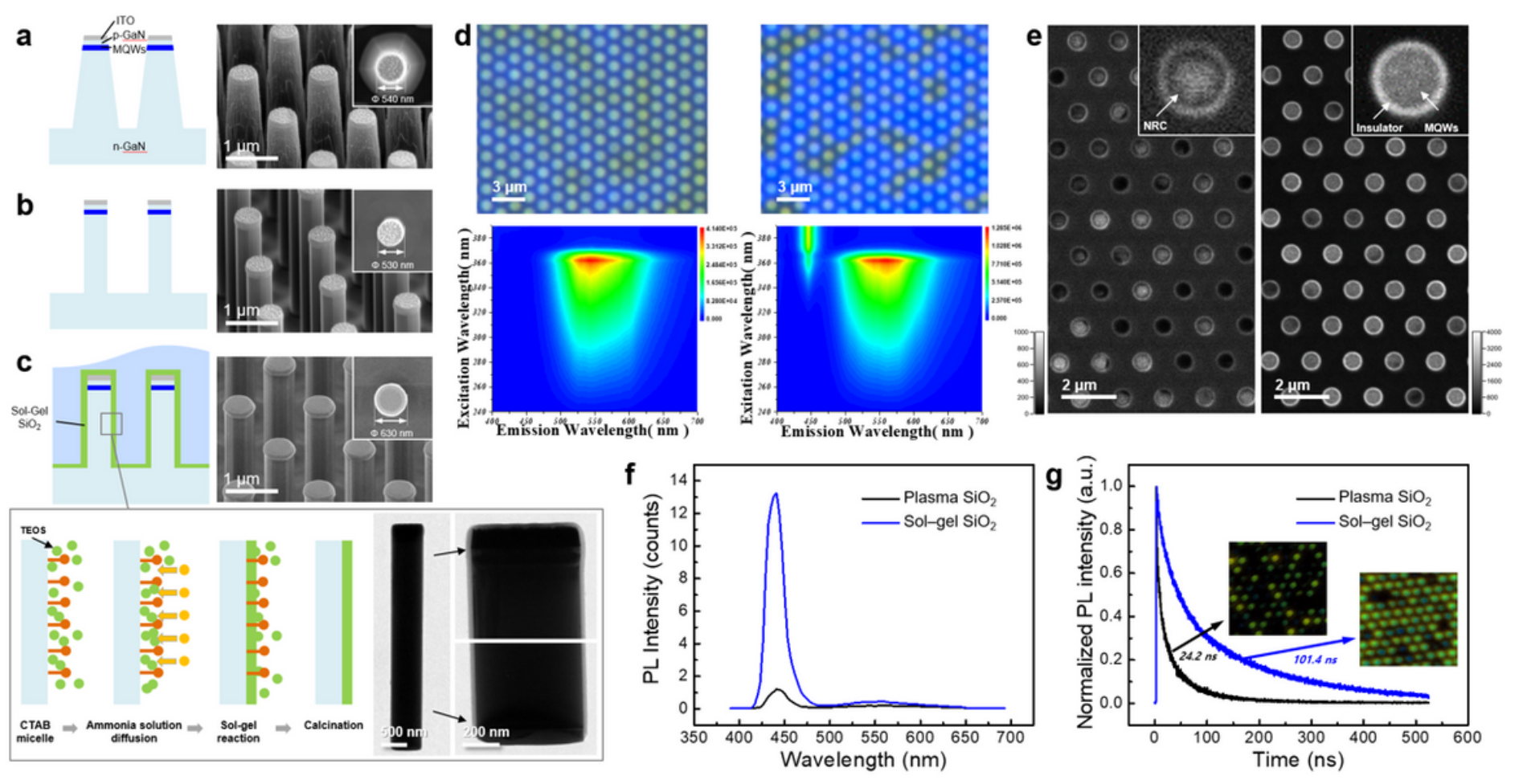

Figure 1

Fabrication of nLEDs and their optical properties. a-c, Schematic and corresponding SEM images of nLEDs fabricated via conventional top-down processing methods. a, Dry etching, $b$, wet etching, and $c$, deposition of SiO2 surface passivation layer via sol-gel method. Inset is a schematic of the sol-gel reaction on the GaN LED nanorod. d, PL image (top: blue and yellow emission are co-displayed) and fluorescence excitation-emission spectra (down) of nanorods with plasma enhanced ALD SiO2 passivation (left) and sol-gel SiO2 passivation (right). e, Panchromatic CL images of the sub-micron LED rod array on wafer with plasma enhanced ALD SiO2 (left) and sol-gel SiO2 (right) $(\lambda=300-700 \mathrm{~nm})$. $\mathrm{f}, \mathrm{PL}$ spectra and g, PL decay traces of the LED nanorods averaged over the area indicated in the inset images. 
a

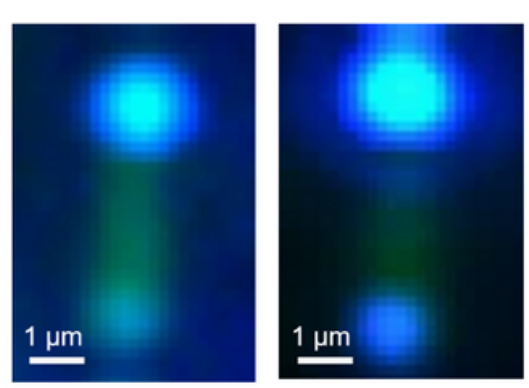

d

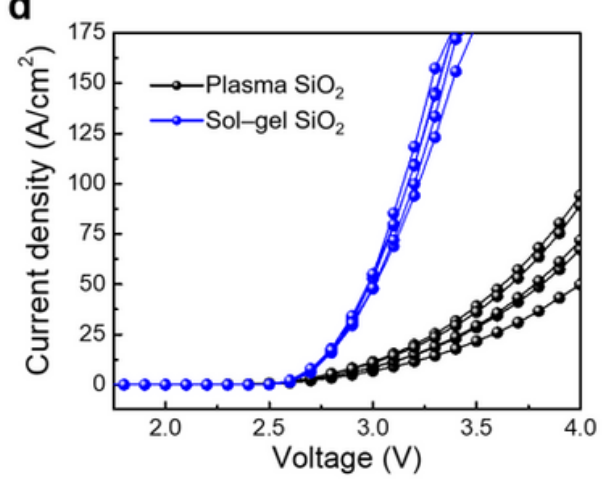

b

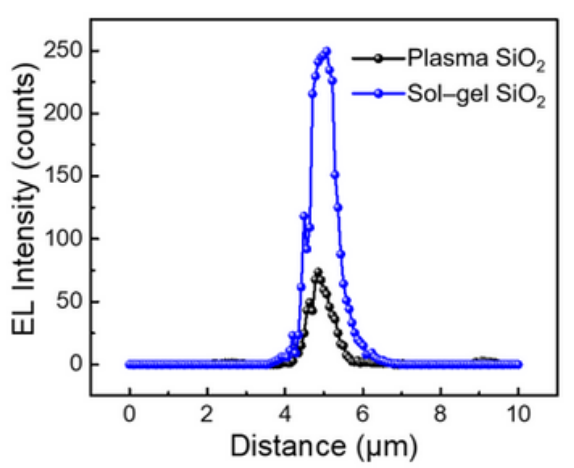

e

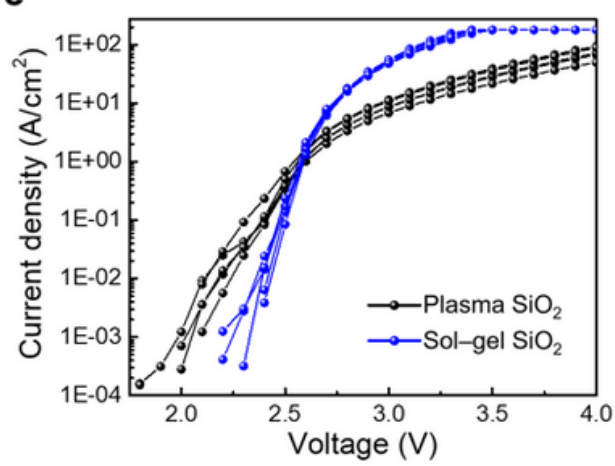

C

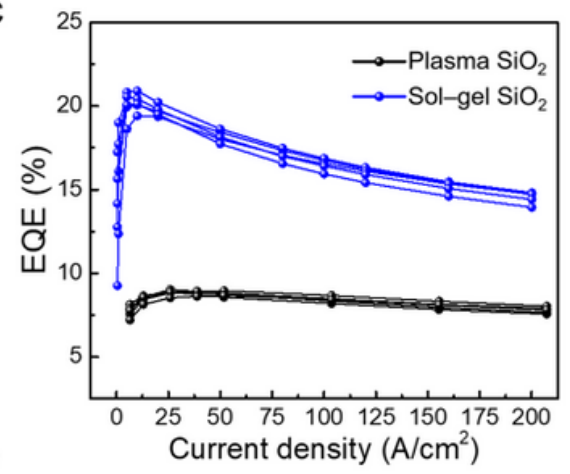

f

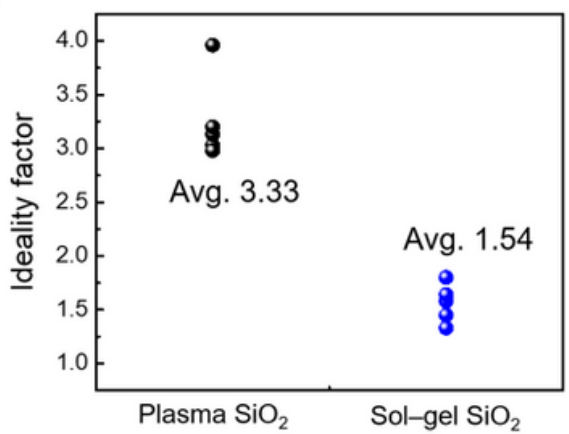

Figure 2

Variation in electroluminescence and current density-voltage $(J-V)$ curves of nLEDs according to the surface passivation method: plasma enhanced ALD- and sol-gel SiO2 deposition. a, EL and PL composite image of a single rod within a pixel based on passivation type: ALD SiO2 (left) and sol-gel $\mathrm{SiO} 2$ (right). b, EL intensity profile across the MQWs in the horizontal direction in Fig. 2a. c, EQE curves for the nLEDs. Each curve is obtained from 60 pixels, with each pixel comprising 6 and 9 rods for ALD SiO2and sol-gel SiO2-passivation, respectively. Current density and voltage characteristics of the nLEDs in d, linear scale and e, log scale. f, Ideality factors obtained from the $\mathrm{J}-\mathrm{V}$ curves at $2.5 \mathrm{~V}$.
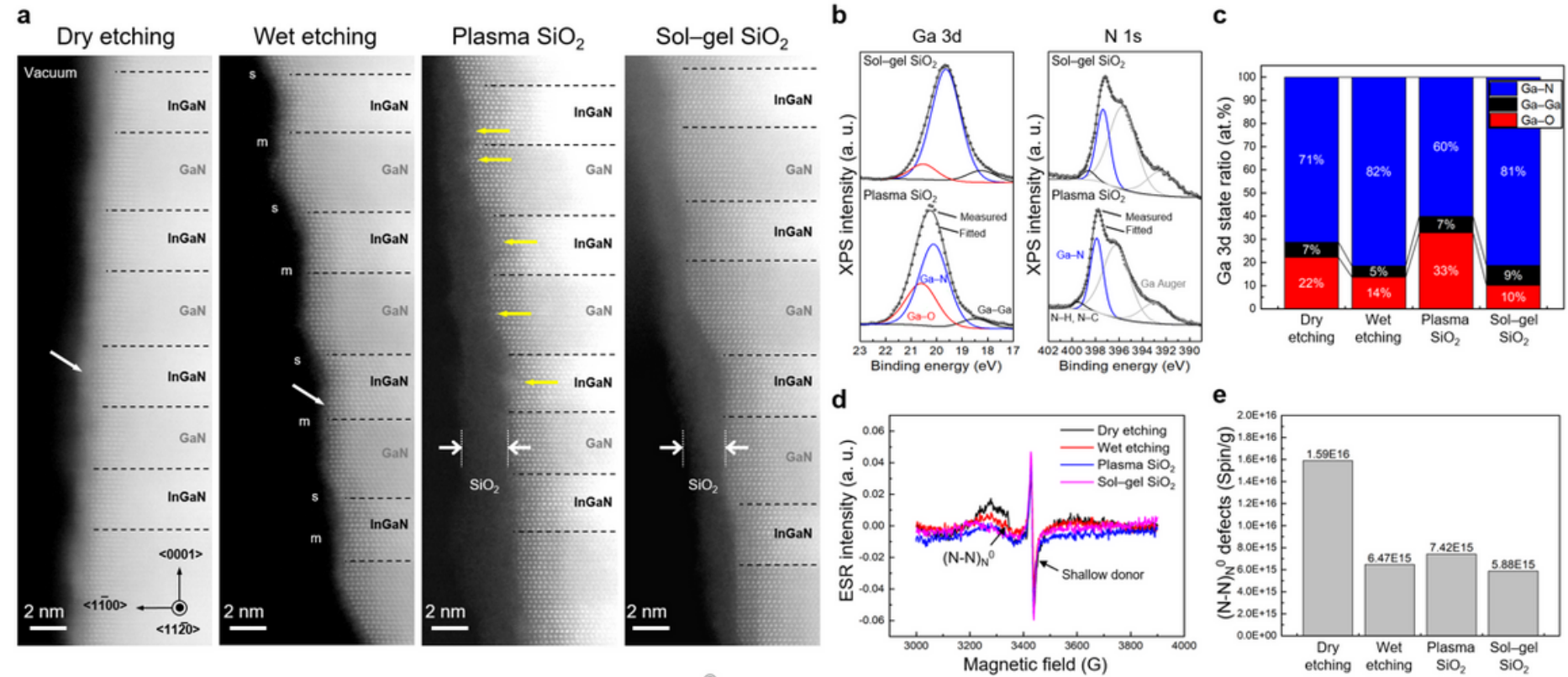


\section{Figure 3}

Surface analysis of the nLEDs after each fabrication step. a, high-angle annular dark-field scanning transmission electron microscopy (HAADF-STEM) images of the sidewall in MQWs according to the fabrication step. The white arrow represents the amorphization of the dry-etched surface. The yellow arrows indicate the plasma damage concentrated on the sidewall of the InGaN QWs. b, XPS core level spectra of nLEDs with a $2 \mathrm{~nm}$ thick SiO2 coating: Ga 3d (left), N 1s (right) c, Ga 3d state ratios obtained from the XPS spectra. d, ESR spectra of the nanorods after each fabrication step. e, N-N split interstitials of the nanorods based on the ESR spectra.

a

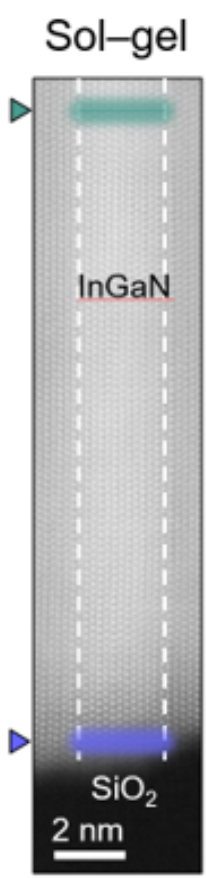

b

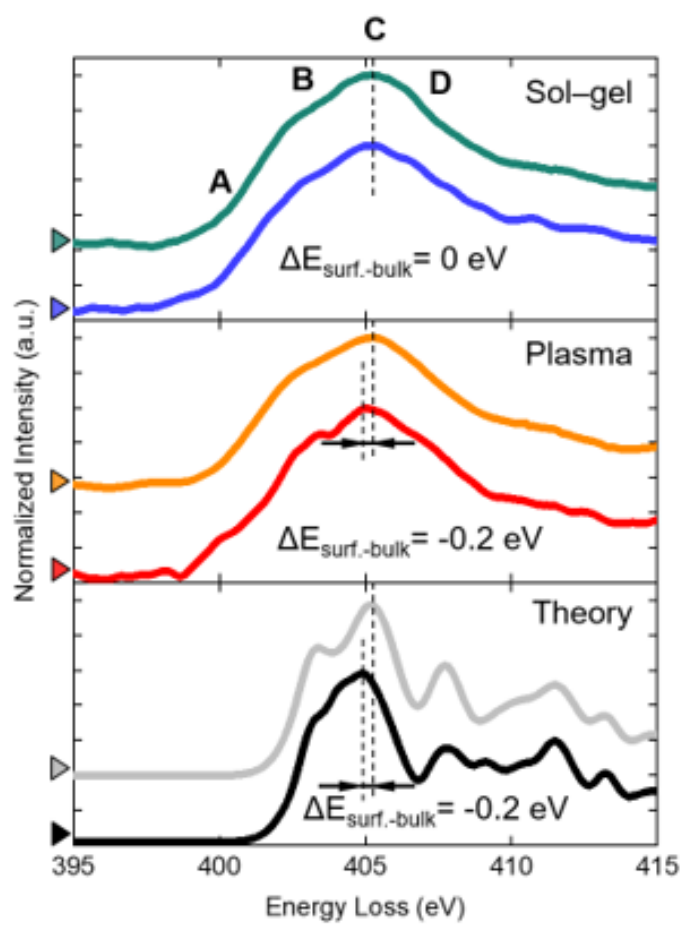

Bulk

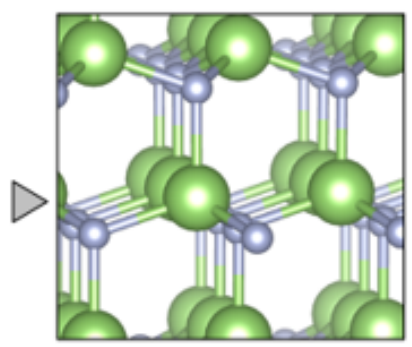

$\mathrm{H} \quad \mathrm{V}_{\mathrm{Ga}}-\mathrm{O}_{\mathrm{N}}-2 \mathrm{H}$

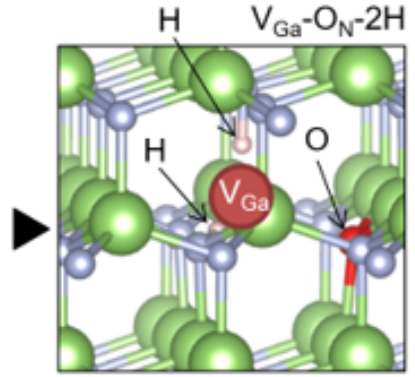

\section{Figure 4}

Defects in the sidewalls of InGaN QWs fabricated via different passivation methods. a, HR-STEM images of the InGaN QWs. Each coloured layer indicates the region at which the corresponding EEL spectrum was obtained, i.e., bulk (green: sol-gel and yellow: plasma ALD) and surface (blue: sol-gel and red: plasma). b, N-K-edge spectra of the regions indicated in Fig.4a. Spectral features of the N-K ELNES, which were theoretically verified using DFT calculations are shown in the bottom panel.

\section{Supplementary Files}

This is a list of supplementary files associated with this preprint. Click to download.

- ExtendedData.docx 